\title{
Doença de Chagas na Região Norte do Brasil: Análise dos casos no período de 2010 a
}

\section{9}

\author{
Chagas Disease in the North Region of Brazil: Analysis of cases in the period from 2010 to 2019 \\ Enfermedad de Chagas en la Región Norte de Brasil: Análisis de casos en el período de 2010 a 2019
}

Recebido: 23/03/2021 | Revisado: 09/04/2021 | Aceito: 29/04/2021 | Publicado: 14/05/2021

Francisco Cezar Aquino de Moraes

ORCID: https://orcid.org/0000-0003-0623-8135 Universidade Federal do Pará, Brasil

E-mail: francisco.cezar2205@gmail.com

Ellen Sabrinna dos Remédios Passos

ORCID: https://orcid.org/0000-0002-6120-5881 Universidade Federal do Pará, Brasil E-mail: ellensasa09@gmail.com

Paula Moraes Costa

ORCID: https://orcid.org/0000-0002-0628-5882 Universidade Federal do Pará, Brasil E-mail: paulamcosta@ufpa.br

Fernando Rocha Pessoa

ORCID: https://orcid.org/0000-0002-1215-8804 Universidade Federal do Pará, Brasil

E-mail: fernandopessoarocha@gmail.com

Leidiana de Jesus da Silva Lopes

ORCID: https://orcid.org/0000-0002-9301-5206

Universidade Federal do Pará, Brasil E-mail: psleidiana@yahoo.com.br

\begin{abstract}
Resumo
O presente artigo tem por objetivo apresentar dados referentes a Região Norte (RN) do Brasil quanto ao número de casos novos de Doença de Chagas Aguda (DCA) entre 2010 a 2019, nos estados que constituintes (Acre, Amazonas, Amapá, Pará, Rondônia, Roraima e Tocantins), correlacionados com fatores sociais que atuam para a persistência da enfermidade em determinadas localidades. Trata-se de um estudo do tipo descritivo, qualitativo e quantitativo. Foram coletados, analisados e organizados dados referentes ao número de casos de DCA pelas cinco regiões do Brasil (Centro-oeste, Nordeste, Norte, Sudeste e Sul) no período de 2010 a 2019, com enfoque nos estados que compõe a RN, por meio do tabulador que opera na internet (TABNET) do Departamento de Informática do Sistema Único de Saúde (DATASUS). Os dados coletados de Doença de Chagas (DC) nas diferentes regiões brasileiras apontaram que o maior número de casos novos da DC no período de 2010 a 2019 concentravam-se na RN, com expressivo número dos incidentes localizados no estado do Pará. Entre os notificados 54,27\% dos diagnosticados foram do sexo masculino, a escolaridade na maioria dos casos foi informada como ignorada $(91,67 \%)$ e o modo de infecção mais provável foi a oral com 78,54\%. Nesse sentido, A DC ainda ameaça basicamente as regiões mais vulneráveis da América Latina. Torna-se, portanto, de extrema importância a promoção de ações públicas que visem atenuar esse problema de saúde pública, tendo base a equidade e promoção de qualidade de vida para a população.
\end{abstract}

Palavras-chave: Doença de Chagas; Epidemiologia; Perfil de saúde; América latina.

\begin{abstract}
This article aims to present data referring to the North Region (NR) of Brazil regarding the number of new cases of Acute Chagas Disease (ACD) between 2010 to 2019, in the constituent states (Acre, Amazonas, Amapá, Pará, Rondônia, Roraima, and the Tocantins), correlated with social factors that act for the persistence of the disease in certain locations. This is a descriptive, qualitative and quantitative study. Data were collected, analyzed, and organized regarding the number of cases of ACD across the five regions of Brazil (Midwest, Northeast, North, Southeast, and South) in the period from 2010 to 2019, focusing on the states that make up the NR, through of the tab that operates on the internet (TABNET) of the Informatics Department of the Unified Health System (DATASUS). The data collected from Chagas Disease (CD) in different Brazilian regions showed that the largest number of new cases of CD in the period from 2010 to 2019 were concentrated in NR, with a significant number of incidents located in the state of Pará. 54.27\% of those diagnosed were male, education in most cases was reported as ignored (91.67\%) and the most likely mode of infection was oral, with $78.54 \%$. In this sense, DC still basically threatens the most
\end{abstract}


vulnerable regions in Latin America. It is, therefore, extremely important to promote public actions aimed at mitigating this public health problem, based on equity and promoting quality of life for the population.

Keywords: Chagas disease; Epidemiology; Health profile; Latin America.

\section{Resumen}

Este artículo tiene como objetivo presentar datos referentes a la Región Norte (RN) de Brasil sobre el número de nuevos casos de Enfermedad de Chagas Aguda (DCA) entre 2010 y 2019, en los estados constituyentes (Acre, Amazonas, Amapá, Pará, Rondônia, Roraima y Tocantins), correlacionados con factores sociales que actúan para la persistencia de la enfermedad en determinadas localizaciones. Se trata de un estudio descriptivo, cualitativo y cuantitativo. Se recopilaron, analizaron y organizaron datos sobre el número de casos de DCA en las cinco regiones de Brasil (Medio Oeste, Noreste, Norte, Sudeste y Sur) en el período de 2010 a 2019, con un enfoque en los estados que integran la RN, a través de de la pestaña que opera en internet (TABNET) del Departamento de Informática del Sistema Único de Salud (DATASUS). Los datos recolectados de la enfermedad de Chagas (EC) en diferentes regiones brasileñas mostraron que el mayor número de nuevos casos de EC en el período de 2010 a 2019 se concentraron en RN, con un número significativo de incidentes ubicados en el estado de Pará. $54.27 \%$ de los diagnosticados eran hombres, la educación en la mayoría de los casos se informó como ignorada $(91,67 \%)$ y el modo de infección más probable fue la oral, con un $78,54 \%$. En este sentido, la CD sigue siendo una amenaza fundamental para las regiones más vulnerables de América Latina. Por tanto, es de suma importancia promover acciones públicas encaminadas a mitigar este problema de salud pública, basadas en la equidad y la promoción de la calidad de vida de la población.

Palabras clave: Enfermedad de Chagas; Epidemiología; Perfil de salud; América latina.

\section{Introdução}

A Doença de Chagas Humana (DCH), ou tripanossomíase americana, é uma parasitose crônica, cujo agente etiológico é o Trypanossoma cruzi, um protozoário do tipo flagelado, que é normalmente transmitido aos humanos e outros mamíferos por insetos triatomíneos, da família Redviidae, através da picada na pele para a sucção do sangue sendo que, posteriormente, é depositada suas fezes com a forma infectante do parasito, que penetra diretamente pelo local da picada ou através de abrasões na pele ou mucosas (World Health Organization Expert Committee, 1991).

A Doença apresenta um caráter emergente, uma vez que sua maior expressão se encontra em países sul-americanos subdesenvolvidos, como o Brasil, que apresenta um maior número de óbitos entre a população de baixo nível sócioeconômico, adquirindo, portanto, designação de doença negligenciada (Schmunis, 1997).

No Brasil, as pesquisas iniciaram, em 1907, no estado de Minas Gerais, sendo os estudos administrados por Carlos Ribeiro Justiniano das Chagas, assistente do Instituto Manguinhos, comissionado pelo sanitarista Oswaldo Cruz. Primariamente, seu objetivo era saber o que estava havendo com a saúde dos operários que trabalhavam nos setores mineiros. (Dias, Laranja \& Nóbrega, 1945). Assim, Carlos Chagas começou a investigar se havia possibilidade de os insetos triatomíneos, residentes em muitas casas de operários, serem vetores de algum parasita, uma vez que a população local apresentava, frequentemente, arritmias, mortes súbitas e sinais de insuficiência cardíaca (Galvão, 2014). Ao coletar triatomíneos da residência dos indivíduos doentes, o pesquisador mostrou em seus estudos que quase a totalidade dos insetos apresentava um parasito flagelado em seu intestino médio e reto, que foi registrado com o nome Schizotrypanum cruzi, em homenagem a seu mestre Oswaldo Cruz, que atualmente tem o nome de Trypanosoma cruzi (Chagas, 1909; Galvão, 2014).

Os triatomíneos permanecem imóveis durante o dia, escondidos em frestas nas paredes ou em esconderijos entre as telhas, saindo apenas pela noite para se alimentar do sangue humano. No momento da picada, nenhum tipo de dor é sentido, pois a saliva do inseto possui propriedades anestésicas, que potencializam, assim, o processo de sucção sanguínea (Lent \& Wygodzinsky, 1979). Com a criação em laboratório dos insetos, ficou provado que o T. cruzi não passava para a prole larval, sendo, portanto, a aquisição do flagelado através de sua alimentação com um sangue humano ou animal contaminado (Chagas, 1909). Os insetos adultos raramente voam e normalmente não deixam as habitações humanas quando há fontes alimentares suficientes no local. Quanto a sua instalação nas moradias, embora os triatomíneos sejam atraídos pela luz artificial, eles são 
péssimos voadores, sendo, então, a principal via de transporte domiciliar do inseto através do próprio homem, que levam os ovos dos triatomíneos em roupas e diversos utensílios e objetos pessoais (Lent \& Wygodzinsky, 1979).

De acordo com Chagas (1916), os indivíduos que adquirem a Doença de Chagas (DC), na sua fase aguda, podem ser distinguidos em dois grandes grupos, cujo critério principal da distinção seria a expressão da sintomatologia e prognóstico. Assim, um grupo, segundo ele, teria a forma benigna, por conta de os sintomas serem mais brandos, havendo, então, maior possibilidade de sobrevida, que evoluirá, posteriormente, para a forma crônica da doença. Já o outro grupo teria a forma aguda grave ou meningoencefálica, que apresenta como características processos inflamatórios nos centros nervoso, sendo os pacientes desse grupo mais suscetíveis a letalidade da DCH.

O período de incubação da parasitose, assintomático, é, em média, de 8 a 12 dias após a infecção por T. cruzi. Na fase aguda, o paciente pode ou não apresentar lesão(s) na pele que indique a porta de entrada do parasita, possuindo característica furunculoide quando na pele, enquanto no olho, é o típico achado clínico denominado de "sinal de Romaña", em que conjuntivas e pálpebras de um dos olhos mostram-se edemaciadas (Lent \& Wygodzinsky, 1979).

A fase crônica é caracterizada pela cardiopatia chagásica ou por quadros de megaesôfago e megacólon (Costa, Tavares, Aquino \& Moreira, 2013). É importante frisar que pacientes crônicos são, na maioria das vezes, casos agudos que não passaram por tratamento (Meyer, Kaneshima \& de Souza-Kaneshima, 2006; Costa et al., 2013).

A Doença de Chagas apresenta eficácia para cura próxima a 100\% com administração dos fármacos benznidazol e nifurtimox logo após a infecção por $T$. cruzi, sendo perdida gradualmente sua ação terapêutica a medida que se é utilizado em um estágio mais tardio da doença, com aumento significativo de efeitos adversos, especialmente em pacientes de maior idade (World Health Organization, 2020).

A DC apresenta ampla distribuição geográfica, que inclui os possíveis reservatórios e vetores, cobrindo desde a porção sul dos Estados Unidos seguindo ao sul da Argentina e Chile, na América do Sul. Assim sendo, todas as Américas estão presentes na área de abrangência, sofrendo com a exposição a uma possível infecção por T. cruzi (Coura \& Dias, 2009). Estima-se que 6 a 7 milhões de pessoas no mundo, sendo a maioria na América Latina, estejam infectadas com o parasito causador da DCH, sendo a transmissão do tipo vetorial, isto é, aquela passada através do triatomíneo, a mais prevalente (World Health Organization, 2020).

A DCH já foi inteiramente confinada a ambientes rurais, principalmente na América Latina. Contudo, na modernidade, a crescente migração dos indivíduos desses locais para os centros urbanos, somando-se a uma crescente urbanização acelerada e desordenada, acarretou, como consequência, que os casos da doença tivessem um registro majoritariamente urbano (World Health Organization, 2020). Schmunis (1997) aponta para a consequência danosa do aumento da carga sobre o sistema de saúde urbano latino-americano devido a doença, uma vez que este já era rotineiramente sobrecarregado, o que gera, além do aumento das despesas governamentais, uma diminuição significativa na qualidade da prestação dos serviços de saúde.

Por se tratar de uma zoonose, o seu número de reservatórios animais é um grande fator de influência, a DCH não pode ser erradicada. Por não possuir vacina ou medicação específica para a utilização em indivíduos que contraem a doença, uma intervenção no ambiente em que o vetor possivelmente poderá colonizar é de extrema importância (World Health Organization Expert Committee, 2002). Pôr os triatomas serem hematófagos obrigatórios, eles procuram a proximidade com o homem (e outros animais, especialmente os domésticos), sendo as habitações mais rústicas de barro, denominada popularmente de "cafuás" o local mais propício para procriação do inseto, que se escondem no interstício das paredes ou outros esconderijos nos arredores, saindo apenas pela noite, para se alimentar (Dias et al., 1945).

A utilização de diferentes inseticidas para eliminar o vetor da doença constitui, atrelado a uma boa educação sanitária por parte da população, um importante fator de diminuição efetiva do índice de infestação domiciliar, bem como o 
desenvolvimento da $\mathrm{DCH}$, embora a melhora das condições de moradia, mesmo as mais pequenas como o reboque das paredes e mudança do teto, mostrem resultados ainda melhores (Schmunis, 1997).

O presente artigo tem por objetivo apresentar dados referentes a Região Norte do Brasil quanto ao número de casos novos de Doença de Chagas Aguda (DCA) no período de 2010 a 2019, nos estados que a constituem, correlacionando com fatores sociais que atuam para a persistência da enfermidade em determinadas localidades.

\section{Metodologia}

Trata de um estudo do tipo descritivo, qualitativo e quantitativo. Foram coletados dados referentes ao número de casos de Doença de Chagas Aguda (DCA) notificados pelas cinco regiões do Brasil: Centro-oeste, Nordeste, Norte, Sudeste e Sul, no período de 2010 a 2019 no Departamento de Informática do Sistema Único de Saúde (DATASUS), por meio do tabulador que opera na internet (TABNET).

A partir dos dados coletados, foram analisadas as variáveis: sexo, faixa etária, evolução da doença, escolaridade e modo provável de infecção nos Estados que constituem a região com maior número de casos, Região Norte (RN) do país, que é composta pelos estados do Acre (AC), Amazonas (AM), Amapá (AP), Pará (PA), Rondônia (RO), Roraima (RR) e Tocantins (TO). As informações organizadas em gráficos foram elaboradas através do software BioEstat 5.3.

Por ser um estudo desenvolvido a partir de dados secundários, divulgado de forma pública pelas fontes supracitadas, a pesquisa dispensa o aceite do Comitê de Ética em Pesquisa, segundo resolução número 580/2018 do Conselho Nacional de Saúde.

\section{Resultados}

Os dados coletados de DC nas diferentes regiões brasileiras, apontou que o maior número de casos novos da doença de DC nas diferentes regiões brasileiras no período de 2010 a 2019, concentravam-se majoritariamente na Região Norte (RN) do Brasil. Nesses 9 anos, o Brasil apresentou um total de 2.482 casos novos da parasitose em todo o território nacional, sendo

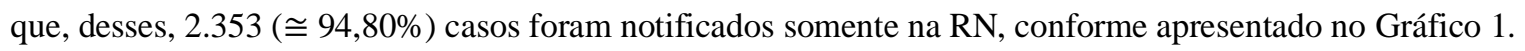


Gráfico 1: Notificação de DC nas Regiões do Brasil, no período de 2010 a 2019.

\section{Novos casos de Doença de Chagas notificados nas Regiões do Brasil de 2010 a} 2019

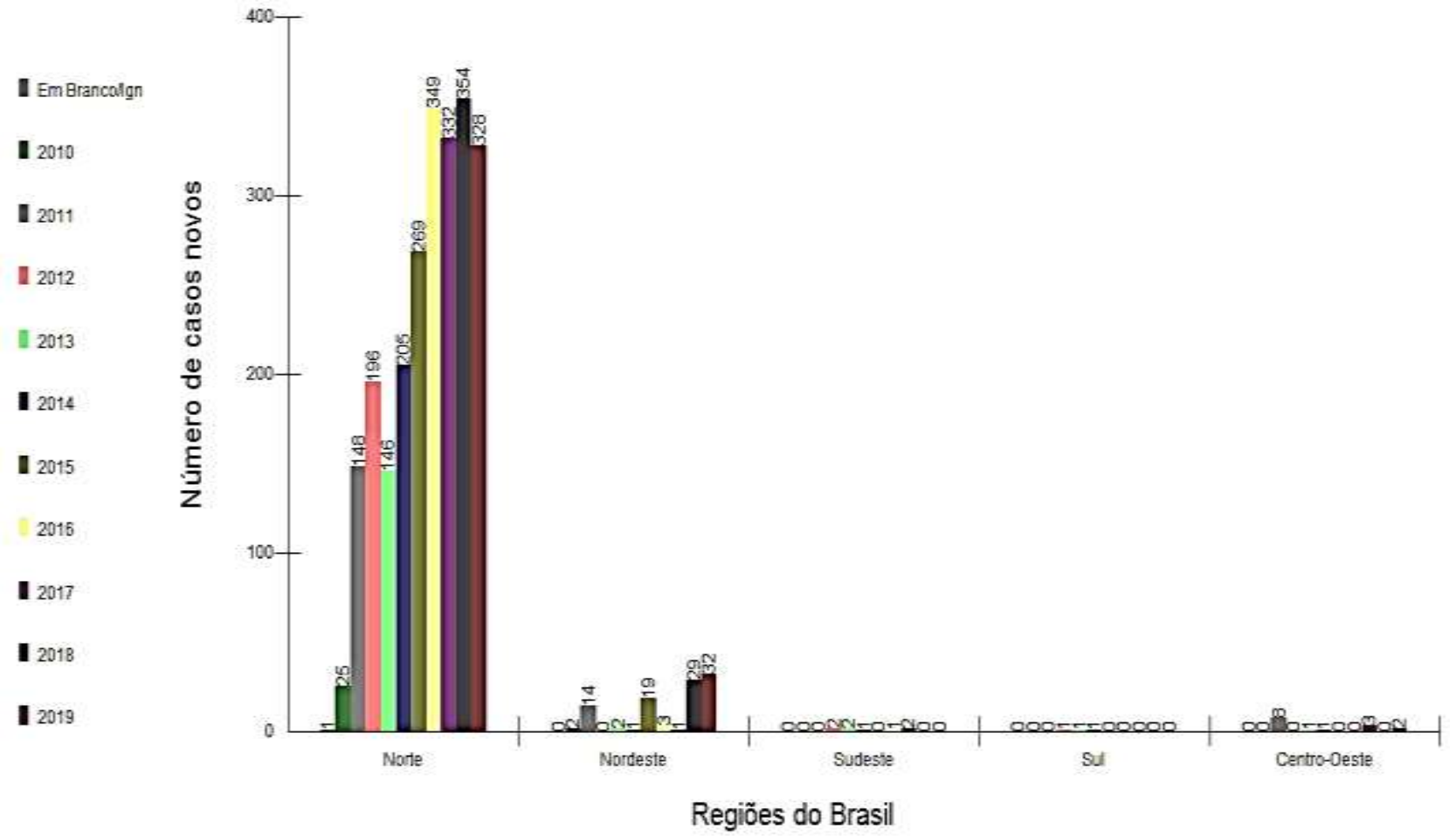

Fonte: Autores. Dados epidemiológicos retirados do Departamento de Informática Informação do Sistema Único de Saúde- DATASUS, Brasil (2020).

Dentre os estados que constituem a RN, o Pará é o que apresenta o maior número de casos de DC aguda, como mostra o Gráfico 2, não só em relação aos outros estados do Norte, mas também a todos do Brasil, nos anos de 2010 a 2019.

No referido período, o Estado do Pará apresentou um total de 1.990 (mil novecentos e noventa) notificações de DCA, seguido pelo Amapá, com 140 casos. Nesse sentido, analisar a sua especificidade, disposição e representação, atrelado a uma comparação analítica e crítica constitui um fator de grande importância para a compreensão da condição peculiar da localidade. 
Gráfico 2: Notificação de casos de DC nos estados brasileiros que constituem a Região Norte:

\section{Casos de Doença de Chagas notificados na Região Norte do Brasil}

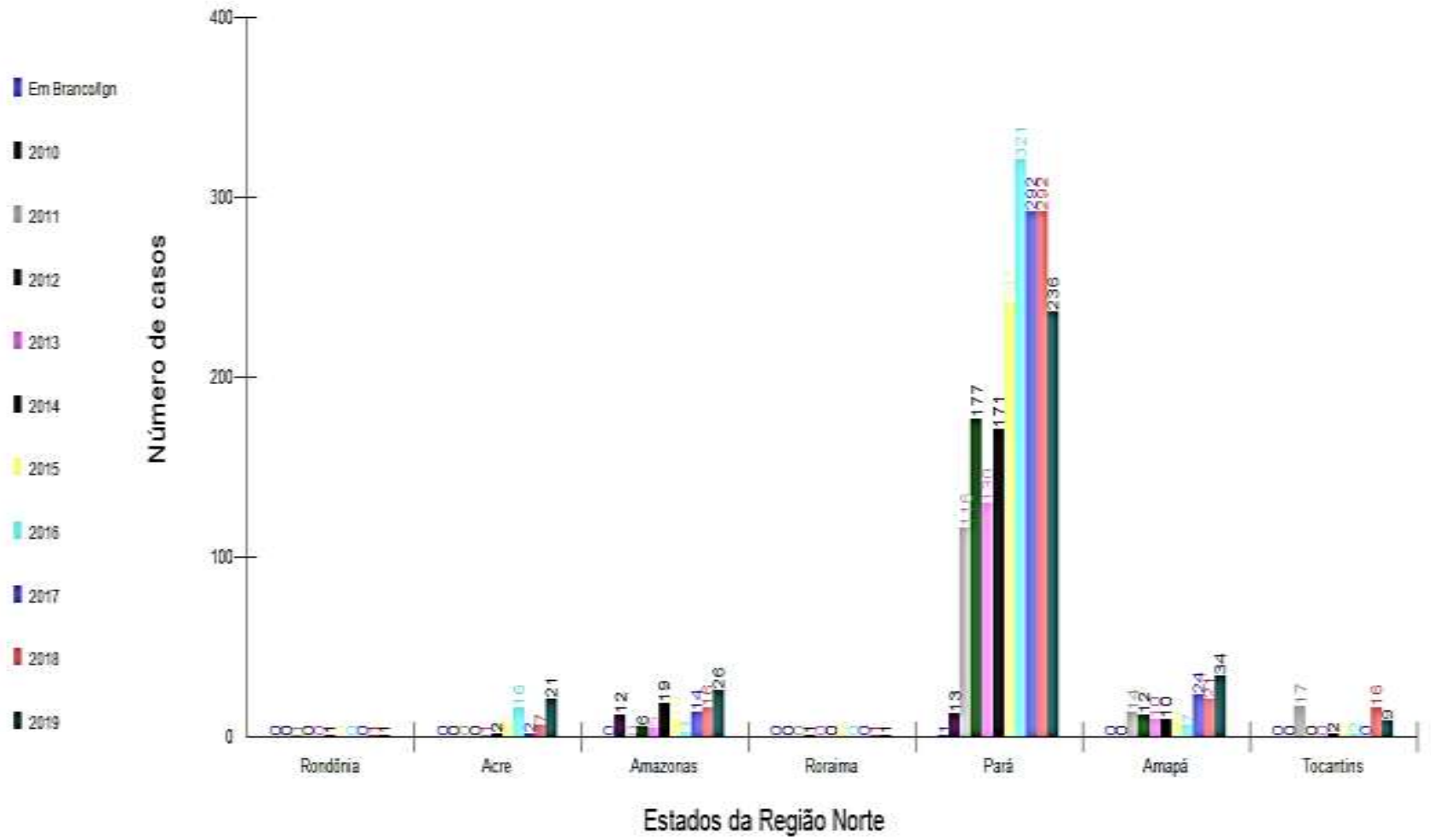

Fonte: Autores. Dados epidemiológicos retirados do Departamento de Informática Informação do Sistema Único de Saúde- DATASUS, Brasil (2020).

Durante o período de 2010 a 2019 foram diagnosticados 2.353 casos de DC na Região Norte. Entre os notificados $54,27 \%$ dos diagnosticados foram do sexo masculino e a escolaridade na maioria dos casos foi informada como ignorada (91,67\%). Os dados analisados apontam que o modo de infecção mais provável foi a oral com 78,54\% dos casos e que em 86,78\% dos casos a enfermidade foi diagnosticada em pessoas vivas, conforme podemos observar no Quadro 1. 
Quadro 1: Características sociodemográficas dos casos de doença de Chagas aguda, Norte, 2010 a 2019.

\begin{tabular}{|c|c|c|}
\hline Variáveis (n) & $\mathbf{n}$ & $\%$ \\
\hline \multicolumn{3}{|l|}{ Sexo } \\
\hline Masculino & 1277 & 54,27 \\
\hline Feminino & 1076 & 45,73 \\
\hline \multicolumn{3}{|l|}{ Escolaridade } \\
\hline Ignorada & 2157 & 91,67 \\
\hline Nenhuma & 196 & 8,33 \\
\hline \multicolumn{3}{|l|}{ Modo Provável de Infecção } \\
\hline Ignorada & 318 & 13,51 \\
\hline Vetorial & 177 & 7,52 \\
\hline Vertical & 3 & 0,13 \\
\hline Acidental & 4 & 0,17 \\
\hline Oral & 1848 & 78,54 \\
\hline Outro & 3 & 0,13 \\
\hline \multicolumn{3}{|l|}{ Evolução da Doença } \\
\hline Ignorada & 272 & 11,57 \\
\hline Vivo & 2042 & 86,78 \\
\hline Óbito pelo agravo notificado & 33 & 1,40 \\
\hline Óbito por outra coisa & 6 & 0,25 \\
\hline
\end{tabular}

(n): Número total de casos notificados. Fonte: Autores. Ministério da Saúde/SVS - Sistema de Informação de Agravos de Notificação - Sinan Net, Brasil (2020).

Ainda sobre os dados analisados na Região Norte, observou-se que pessoas na faixa etária de 20 a 39 anos foram as mais acometidas (33,65\%), seguidas das de 40 a 59 anos (24,10\%). Observa-se o detalhamento das informações no Gráfico 3.

Gráfico 3: Notificação de casos por faixa etária na Região Norte no período de 2010 a 2019.

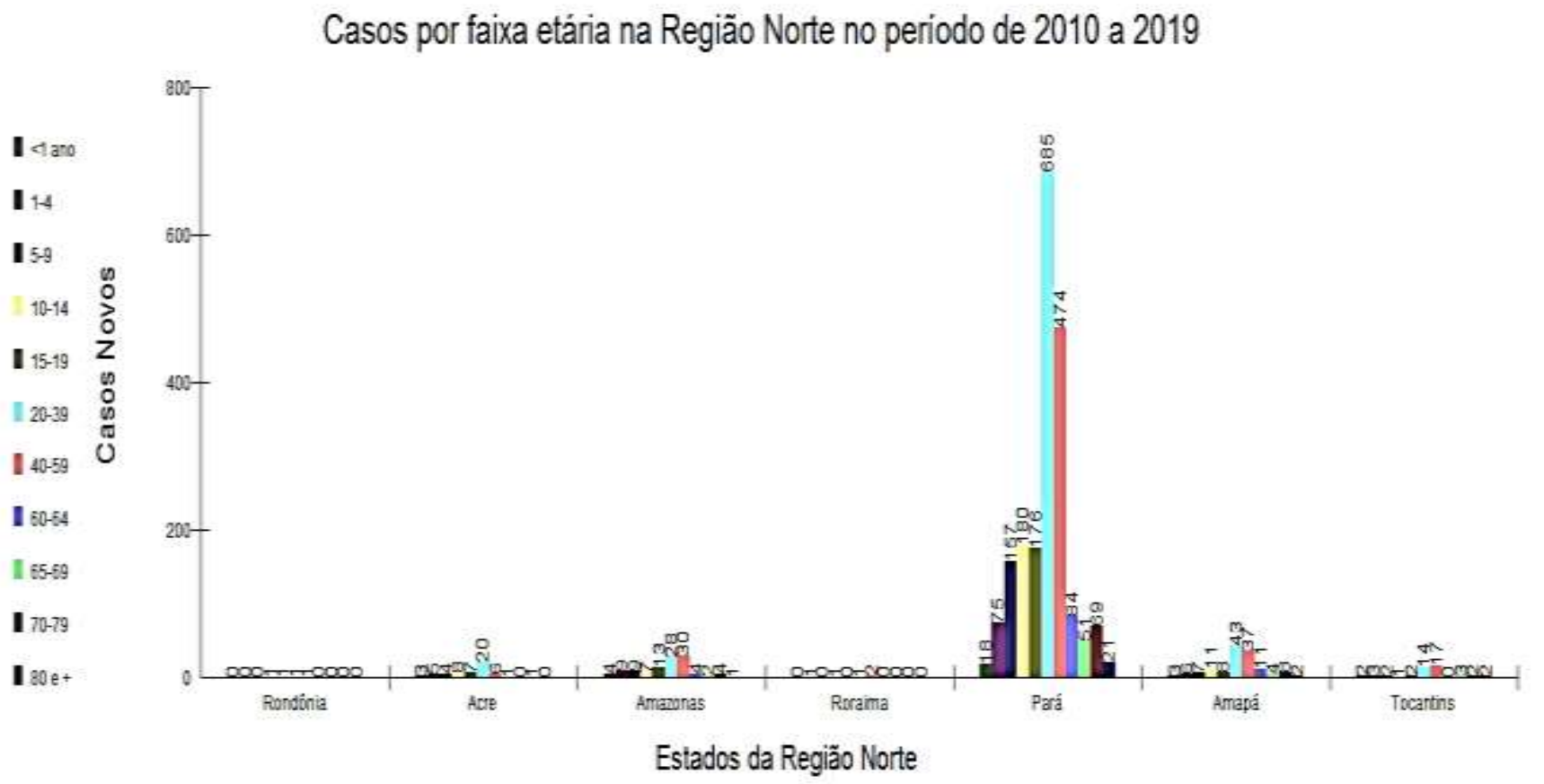

Fonte: Autores. Dados epidemiológicos retirados do Departamento de Informática Informação do Sistema Único de Saúde- DATASUS, Brasil (2020). 


\section{Discussão}

Os dados apontam que a região Norte do Brasil é a que possuiu 94,80\% do total de casos notificados no período estudado, um quantitativo expressivo que representa uma preocupação em relação à saúde de nortistas. Na RN do Brasil, a aquisição da doença chagásica de forma passiva, por meio da transmissão oral, veio ganhando significativa importância nos últimos anos, uma vez que microepidemias estavam associados ao consumo de polpas de açaí contaminadas seja pela contaminação do fruto ou da polpa em si, através de dejetos de animais reservatórios ou dos insetos vetores infectados pelo parasito (Labello Barbosa, 2010; Passos et al., 2012). Nesse novo tipo de transmissão, a ingestão, por via oral, das formas tripomastigotas metacíclicas do parasito gera, como consequência, um quadro agudo da enfermidade que muitas vezes evolui para o óbito (Camandaroba, Lima \& Andrade, 2002).

Embora grande parte da zoonose seja transmitida ao homem majoritariamente por vetores, por via transfusional ou congênita, na atualidade é presenciado uma nova modalidade de infecção por T. cruzi, que é adquirido passivamente, atra vés do consumo de alimentos contaminados (Pérez-Gutiérrez, Agrelo, \& Figueroa, 2006). Labello Barbosa (2010) mostrou em seu trabalho que o T. cruzi pode sobreviver na polpa de açaí, em diferentes temperaturas, sob um período variado, com sua virulência preservada, quando infectados camundongos passivamente, através do consumo da polpa da referida fruta.

Segundo (Farias \& Brito, 2020) o açaí significa para o ribeirinho a sustentabilidade e a base de nutrição, é um fator cultural e uma identidade do extrativismo e consumo na Amazônia. A RN concentra a produção mais expressiva de açaí, sendo o Pará o maior produtor mundial e exportador brasileiro, seguido pelo Amazonas (AM). Os dois estados, juntos, representam 87,5\% do total da produção brasileira de açaí. Vale ressaltar que a DC pouco tem destaque nas políticas públicas dos países afetados, pois atinge na maioria das vezes a população pobre. Assim, o alimento é encontrado em feiras e mercados em estado natural, sem passar pelos processos de lavagem e pasteurização. Desse modo, o número de casos de DC está atrelado com o alto consumo e produção de açaí sem a higiene adequada. É possível estabelecer essa relação, pois $62 \%$ dos locais com o selo “Açaí Bom” feitos pelo Departamento de Vigilância Sanitária de Belém do Pará, capital do Estado, estão em categoria REGULAR e 38\% em categoria RUIM (Companhia Nacional de Abastecimento, 2019; Médicos Sem Fronteiras, 2017; Fundação Oswaldo Cruz, 2010; Labello Barbosa, 2010; Silva, Silva, Sousa \& Sousa, 2017; Dias et al., 2016).

As consequências negativas da globalização são mais evidentes em países emergentes, sendo os países que constituem a América Latina vítimas de fatos como a migração rural-urbana desordenada com ações antrópicas excessivas associadas com invasões de ambientes naturais onde vivem várias espécies de animais que podem transmitir doenças. O T. cruzi, flagelado causador da DC, expõem a marca de uma sociedade que vive cotidianamente em precária realidade socioeconômica e de saúde (Dias, 2007).

Dentre as regiões do Brasil, segundo o IBGE, a Norte é a que apresenta, no ano de 2018, após a região Nordeste, o mais baixo rendimento nominal mensal domiciliar per capita, fato que influencia diretamente na saúde do indivíduo e da sociedade. O segundo menor rendimento nominal mensal per capita explicita as contradições em âmbito sociais, econômicos e de saúde, constituidores intrínsecos dos Determinantes Sociais em Saúde (DSS), que incidem diretamente sobre o indivíduo e a sociedade. Dessa forma, os DSS, que abarcam aspectos sociais, econômicos, culturais, étnicos/raciais, psicológicos e comportamentais que influenciam na saúde e nos fatores de risco que interferem na população, tais como moradia, alimentação, escolaridade, renda e emprego, avaliam as dimensões do processo de saúde dos grupos humanos, tanto do ponto de vista do individual, quanto da coletividade na qual ele é inserido (Buss \& Pellegrini Filho, 2007).

O rendimento domiciliar per capita é uma razão entre o total dos rendimentos domiciliares e o total dos moradores. Sua importância está em analisar a contribuição do local pesquisado na receita nacional. Dentre os estados da RN, o Pará apresenta a renda Nominal Domiciliar per capita de 863 reais, o Amazonas de 791 reais e o Amapá de 857 reais. Esses são os menores números da Renda Nominal Domiciliar per capita do Norte e os maiores registros de casos de DCA de 2010 a 2019. 
Vale ressaltar que estados como Roraima com Renda Nominal Domiciliar per capita de 1204 reais e Rondônia de 1113 reais possuem os menores números de casos da região. Estes por possuírem dados econômicos mais positivos, apresentam populações menos vulneráveis, pois há maior qualidade nos DSS (saúde, alimentação, educação, saneamento) (Instituto Brasileiro de Geografia e Estatística, 2019).

Assim, um baixo acesso a recursos financeiros desencadeia uma sucessão de eventos, tais como, precária condição de vida, educação e saúde, tanto atual quanto futura, o que, diretamente, contribui para a persistência da DC em âmbito regional.

O Brasil é um país subdesenvolvido, com suas dimensões continentais, está passando por fortes transformações no crescimento populacional e econômico, o que implica em uma persistência e intensificação de graves desigualdades socioeconômicas regionais. Doenças associadas ao contexto social de iniquidade presente, somado a negligência governamental afligem parte considerável da população, resultando em situações de vulnerabilidade individual e coletiva que envolvem a Doença de Chagas. Novos problemas de saúde emergem como resultado do intenso processo de urbanização e das mudanças no campo socioambiental, enquanto outros tendem a persistir e coexistir evidenciando a necessidade de possuir ações específicas, ajustadas a novas realidades, da rede de atenção à saúde (Dias et al., 2016).

Outros sinais do comportamento negligente do Estado perante políticas socioeconômicas e de saúde na Região Norte é a presença de cidades, segundo os dados do Ranking de Saneamento do Instituto Trata Brasil de 2020: Ananindeua (PA), Manaus (AM), Porto Velho (RO), Macapá (AP), Santarém (PA) e Belém (PA) entre os dez piores índices de saneamento básico do Brasil. Ademais, vale ressaltar, como um dado consoante a relação diretamente proporcional entre precário saneamento básico e aumento de casos de Doença de Chagas, Ananindeua (PA) foi o que apresentou o maior número de casos notificados da DC, acumulados (2010-2019), quando comparado com todos os outros municípios que constituem cada estado da RN, com um total de 398 casos.

Dessa forma, migrações humanas não controladas, degradação ambiental sem planejamentos, maior concentração da população em áreas urbanas e problemas socioeconômicos inserem-se como determinantes e condicionantes para a transmissão de T. cruzi. Negligenciadas, as populações infectadas apresentam maior vulnerabilidade, que se expressa pela: sobreposição e maior probabilidade de adquirir outras doenças, menor prevenção, menor acesso à rede de serviços de saúde, baixa qualidade da atenção recebida em serviços de atenção primária, menor recepção de tratamentos essenciais, maior expectativa de desenvolver formas graves da doença e maior risco de evolução para óbito. (Dias et al., 2016). Além disso, prevalecem a ocorrência de casos no sexo masculino em relação ao feminino, o que nos leva a inferir que isso se dá pela maior exposição e contato com ambientes que são habitat dos vetores da Doença de Chagas. No que se refere ao acometimento por faixa etária e sexo alguns estudos mostram que a doença não apresenta preferência por faixa etária. Vilhena et al. (2020), em estudo que analisou o perfil clínico e epidemiológico da doença de Chagas aguda nos municípios de Belém, Abaetetuba e Breves, estado do Pará, apontou maior acometimento de casos em pessoas do sexo masculino nos municípios de Abaetetuba e Breves, já em Belém, capital do estado, pessoas do sexo feminino foram as mais acometidas pela doença. O mesmo estudo mostrou que a infecção prevaleceu em adultos na faixa etária de 30 a 59 anos de idade, em dois municípios, Belém e Abaetetuba, já no município de Breves esse maior acometimento foi em pessoas jovens entre 0 e 14 anos.

Vale ressaltar que o fato de a escolaridade dos pacientes ser ignorada mostra uma fragilidade no sistema de vigilância, podendo agravar o quadro dos pacientes infectados e provavelmente pode ser um sinal de dificuldade na prevenção, na maioria das vezes sinais de país com população com baixa classe econômica. O acometimento por DC está associado ao modo de vida, e conhecimento sobre a doença, isto é, costumes em relação a alimentação, moradia, relações sociais e conhecimento básico sobre os modos de infecção são fatores que influenciam no adoecimento dos indivíduos, o que na maioria dos casos se agrava em uma população com a pobreza instalada. Uma melhor saúde está relacionada com o fortalecimento de informação em espaços democráticos para a comunidade. Entretanto, a educação elitista tem como consequência a exclusão social que gera o 
maior desinteresse pela doença, por seu controle e pelas pessoas infectadas (Dias, 2007).

A Vigilância Epidemiológica (VE) já apresentou eficiência no controle da DC nos casos de transmissão vetorial por meio de ações que incluem notificações e visitas domiciliares para análise e dedetização do local, além de orientações. Desse modo, foi nítido o crescimento no número de ações na, para a prevenção e aprimoramento do controle na rede básica de saúde, alicerçada nos princípios da equidade, da universalidade e do controle social. Por isso, vale lembrar também da comunidade como o ator principal para a execução da VE, pois associada ao sistema de saúde, de educação e das instituições de saúde pública - ampliadores de programas de horizontalidade - houve maior eficácia da promoção e controle da doença (Dias, 2000).

\section{Conclusão}

Destarte, a DC ainda ameaça basicamente as regiões pobres da América Latina, isto é, a população que pouco ver acontecer políticas de desenvolvimento, socialmente excluída e pouco alfabetizada. Além disso, o crescimento de notificações durante o período estudado, na região Norte, é um reflexo que a DCA é negligenciada em um local favorável para sua evolução devido aos hábitos e a cultura, ao bioma e às condições sociais desfavoráveis impostas as populações.

O aumento do número de casos gera a necessidade de estruturar uma Vigilância Epidemiológica na região mais eficiente, visando melhorias nas ações de prevenção e controle. Ademais, há a necessidade de qualificação das ações de vigilância em saúde no Sistema Único de Saúde (SUS), por meio de ações de educação permanente, monitoramento e avaliação junto às equipes de saúde.

Os surtos decorrem, geralmente, do consumo de açaí, refletindo uma questão de vigilância sanitária, associada à falta de segurança alimentar e práticas de manipulação de alimentos pouco eficientes. O modo padrão para a investigação seria realizar a análise de alimentos, identificar procedência, local de preparação e de consumo do alimento suspeito e listar todas as pessoas que possam ter ingerido. Nesse cenário, torna-se de extrema importância a promoção de ações públicas que visem atenuar esse problema de saúde pública, tendo base a equidade e promoção de qualidade de vida para a população.

\section{Referências}

Brasil (2021). Sistema de Informação de Agravos de Notificação - SINAN. https://portalsinan.saude.gov.br/.

Buss, P. M., \& Pellegrini Filho, A. (2007). A saúde e seus determinantes sociais. Physis: revista de saúde coletiva, 17, 77-93.

Camandaroba, E. L. P., Pinheiro Lima, C. M., \& Andrade, S. G. (2002). Oral transmission of Chagas disease: importance of Trypanosoma cruzi biodeme in the intragastric experimental infection. Revista do Instituto de Medicina Tropical de São Paulo, 44(2), 97-103.

Chagas, C. (1909). Nova tripanossomíase humana: Estudos sobre a morfologia e o ciclo evolutivo do Schizotrypanum cruzi n. gen., n. sp., agente etiológico de nova entidade mórbida do homem. Memórias do Instituto Oswaldo Cruz, 1(2), 159-218

Chagas, C. (1916). Tripanosomiase americana. Forma aguda da moléstia. Memórias Instituto Oswaldo Cruz, 8(2), 37-60.

Companhia Nacional de Abastecimento (2019). Açaí - Análise Mensal - Março/2019. https://www.conab.gov.br/info-agro/analises-do-mercado-agropecuarioe-extrativista/analises-do-mercado/historico-mensal-de-acai.

Costa, M., Tavares, V., Aquino, M. V., \& Moreira, D. (2013). Doença de chagas: uma revisão bibliográfica. Revista Eletrônica da Faculdade de Ceres, 2(1).

Coura, J. R., \& Dias, J. C. P. (2009). Epidemiology, control and surveillance of Chagas disease: 100 years after its discovery. Memórias do Instituto Oswaldo Cruz, 104, 31-40.

Dias, E., Laranja, F. S., \& Nobrega, G. (1945). Doença de Chagas. Memórias do Instituto Oswaldo Cruz, 43(3), $495-581$.

Dias, J. C. P. (2007). Globalização, iniqüidade e doença de Chagas. Cadernos de Saúde Pública, 23, S13-S22.

Dias, J. C. P. (2000). Vigilância epidemiológica em doença de Chagas. Cadernos de Saúde Pública, 16, S43-S59.

Dias, J. C. P., Prata, A., \& Schofield, C. J. (2002). Doença de Chagas na Amazônia: esboço da situação atual e perspectivas de prevenção. Revista da Sociedade Brasileira de Medicina Tropical, 35(6), 669-678.

Dias, J. C. P., Ramos Jr, A. N., Gontijo, E. D., Luquetti, A., Shikanai-Yasuda, M. A., Coura, J. R., ... \& Alves, R. V. (2016). II Consenso Brasileiro em doença de Chagas, 2015. Epidemiologia e Serviços de Saúde, 25, 7-86. 
Farias, R. T. S., \& Brito, D. M. C. B. (2020). O Açaí Como Referência Sociocultural Para Pensar, Refletir E Construir Conhecimentos Geográficos Nas Escolas Ribeirinhas Da Amazônia Brasileira. Ciência Geográfica, XXIV(2), 833-843.

Fundação Oswaldo Cruz (2010). Açaí contaminado com parasito pode transmitir doença de Chagas! http://www.fiocruz.br/portalchagas/cgi/cgilua.exe/sys/start.htm?infoid=2\&sid=1.

Galvão, C. (2014). Vetores da doença de Chagas no Brasil - Série Zoologia: Guias e Manuais de Identificação. Curitiba: Sociedade Brasileira de Zoologia. $289 \mathrm{pp}$.

Gilber, S. R., \& Soccol, V. T. (2007). Reação em cadeia da Polimerase em comparação com o teste de Imunofluorescência indireta (IFI) e Elisa (Enzimaimunoensaio) no diagnóstico para a doença de Chagas. Dissertação (Mestrado). Universidade Federal do Paraná.

Instituto Brasileiro de Geografia e Estatística (2019). IBGE divulga o Rendimento Domiciliar per capita - RDPC e o Coeficiente de Desequilíbrio Regional CDR 2018. https://biblioteca.ibge.gov.br/visualizacao/periodicos/3095/cdr_2018.pdf.

Instituto Brasileiro de Geografia e Estatística (2019). IBGE divulga o rendimento domiciliar per capita 2018. https://ftp.ibge.gov.br/Trabalho_e_Rendimento/Pesquisa_Nacional_por_Amostra_de_Domicilios_continua/Renda_domiciliar_per_capita/Renda_domiciliar_p er_capita_2018.pdf.

Instituto Nacional de Pesquisas Educacionais Anísio Teixeira (2017). Índice de Desenvolvimento da Educação Básica. http://ideb.inep.gov.br/resultado/

Instituto Nacional de Pesquisas Espaciais (2019). A Estimativa da Taxa de Desmatamento por Corte Raso para a Amazônia Legal em 2019 é de 9.762 km². http://www.inpe.br/noticias/noticia.php?Cod_Noticia=5294.

Instituto Trata Brasil (2019). Ranking do saneamento Instituto Trata Brasil 2020 (SNIS 2018). Go Associados.

Labello Barbosa, R. (2010). Transmissão oral do Trypanosoma cruzi pela polpa de açaí em camundongos. Dissertação (Mestrado). Universidade Estadual de Campinas (UNICAMP).

Lent, H., \& Wygodzinsky, P. (1979). Revision of the Triatominae (Hemiptera, Reduviidae), and their significance as vectors of Chagas' disease. Bulletin of the American museum of Natural History, 163(3), 123-520.

Médicos Sem Fronteiras (2017). Chagas, doença invisível, população negligenciada. https://www.msf.org.br/opiniao/chagas-doenca-invisivel-populacaonegligenciada.

Meyer, I. F., Kaneshima, E. N., \& de Souza-Kaneshima, A. M. (2006). Alterações no sistema digestivo desencadeadas pelo quadro infeccioso do Trypanosoma Cruzi. Iniciação Cientifica Cesumar, 8(1), 11-23.

Passos, L. A. C., Guaraldo, A. M. A., Barbosa, R. L., Dias, V. L., Pereira, K. S., Schmidt, F. L., \& Alves, D. P. (2012). Sobrevivência e infectividade do Trypanosoma cruzi na polpa de açaí: estudo in vitro e in vivo. Epidemiologia e serviços de Saúde, 21(2), 223-232.

Pérez-Gutiérrez, E., Agrelo, R. S., \& Figueroa, R. (2006). Consulta técnica em epidemiologia, prevenção e manejo da transmissão da doença de Chagas como doença transmitida por alimentos. Revista da Sociedade Brasileira de Medicina Tropical, 39(5), 512-514.

Schmunis, G. A. (1997). Tripanossomíase americana: seu impacto nas Américas e perspectivas de eliminação. Clínica e Terapêutica da Doença de Chagas. Um Manual Prático para o Clínico Geral, 11-24.

Silva, F. S., Silva, A. F. M., Sousa, C. L., \& Sousa, J. (2017). Avaliação higiênico-sanitária dos estabelecimentos com selo "Açaí Bom" da Vigilância Sanitária. Brazilian Journal of Food Research, Campo Mourão, 8(4), 157-169.

Teixeira, R. B., \& Oliveira, S. M. (2015). Perfil de pacientes portadores de doença de Chagas em Rio Branco, Acre, Brasil. Rev Soc Bras Clin Med, 13(4), $262-265$.

Vilhena, A. O., Pereira, W. M. M., de Oliveira, S. S., Fonseca, P. F. L., Ferreira, M. S., da Costa Oliveira, T. N., \& de Lima, P. D. L. (2020). Doença de Chagas aguda no estado do Pará, Brasil: série histórica de aspectos clínicos e epidemiológico em três municípios, no período de 2007 a 2015 . Revista PanAmazônica de Saúde, 11,11-11.

World Health Organization (2020). Chagas disease (also known as American trypanosomiasis). https://www.who.int/news-room/fact-sheets/detail/chagasdisease-(american-trypanosomiasis).

World Health Organization Expert Committee. (1991). Control of Chagas disease: report of a WHO expert committee [meeting held in Buenos Aires from 16 to 20 October 1989. World Health Organization technical report series, 811 , i.

World Health Organization Expert Committee. (2002). Control of Chagas disease. World Health Organization technical report series, 905, i. 